\title{
Le travail autonome économiquement dépendant en Espagne
}

\section{Esther Guerrero Vizuete}

\section{(2) OpenEdition}

1 Journals

\section{Édition électronique}

URL : https://journals.openedition.org/rdctss/1655

DOI : 10.4000/rdctss. 1655

ISSN : 2262-9815

Éditeur

Centre de droit comparé du travail et de la sécurité sociale

\section{Édition imprimée}

Date de publication : 1 avril 2019

Pagination : 98-109

ISSN : 2117-4350

\section{Référence électronique}

Esther Guerrero Vizuete, "Le travail autonome économiquement dépendant en Espagne ", Revue de droit comparé du travail et de la sécurité sociale [En ligne], 1 | 2019, mis en ligne le 01 novembre 2021, consulté le 13 novembre 2021. URL : http://journals.openedition.org/rdctss/1655 ; DOI : https:// doi.org/10.4000/rdctss. 1655

\section{(c) (†) $९$}

Revue de droit comparé du travail et de la sécurité sociale est mise à disposition selon les termes de la Licence Creative Commons Attribution - Pas d'Utilisation Commerciale - Pas de Modification 4.0 International. 


\title{
LE TRAVAIL AUTONOME ÉCONOMIQUEMENT DÉPENDANT EN ESPAGNE
}

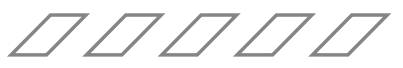

\section{RÉSUMÉ}

L'existence de travailleurs qui développent leur activité professionnelle en étant soumis à la fois à une indépendance juridique comme à une subordination économique n'est pas une situation inhabituelle pour le système espagnol. En effet, la loi 20/2007 du 11 juillet, définit le régime juridique auquel ces travailleurs sont soumis. Pourtant, onze ans plus tard, on peut estimer que cette règle n'a pas répondu aux attentes en omettant de fournir une protection juridique adéquate à cette catégorie de travailleurs.

MOTS CLÉS : Travail indépendant, subordination économique, statut du travail indépendant.

\begin{abstract}
The workers' existence that they were developing his professional activity submitted both to a juridical independence and to an economic subordination was not a situation not known by the Spanish law. His lack of regulation ends with the Law 20/2007 of July 11, in that there is defined the juridical regime to which these workers must remain submitted, though, eleven years later, we can say that this norm has not met with the expectations generated on not having offered them a suitable juridical protection.
\end{abstract}

KEYWORDS : Autonomous Worker, Economic Subordination, Statute of Autonomous Labour. 


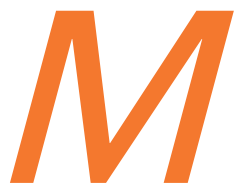
espagnol, comme le montre le fait qu'au 31 décembre 2018, seuls 9497 travailleurs étaient enregistrés comme travailleurs indépendants économiquement dépendants (TRADE) régis par ladite loi ${ }^{1}$.

Lorsqu'il s'est penché sur la manière dont cette nouvelle catégorie de travailleurs devait être régulée, le législateur a été influencé principalement par deux facteurs. La sous-traitance des activités productives occupait une place prépondérante dans les entreprises qui recouraient au travail autonome car cela leur permettait de bénéficier des services de professionnels, flexibles d'un point de vue organisationnel et dotés d'un haut degré d'efficacité économique d'une part et d'autre part, cela leur a permis de devenir des sociétés modulaires, c'est-à-dire des organisations dont la structure peut croître ou diminuer en fonction des besoins de la production. Les travailleurs concernés ont réagi aux changements objectifs et fonctionnels des entreprises ayant pour effet immédiat la flexibilisation du marché du travail. En période de crise, ceci a entraîné des changements dans les modalités d'embauche, plaçant ces travailleurs dans la position de travailleur indépendant au lieu de travailleur salarié. Ainsi, de nouvelles situations sont apparues pour lesquelles le système juridique n'a apporté aucune réponse en termes de réglementation.

Ce phénomène n'a pas seulement conduit à une revitalisation du travail autonome; il a également révélé les lacunes de la protection juridique de ces travailleurs. La combinaison de ces deux facteurs a engendré un grand nombre de situations frauduleuses et le fait que le concept de «faux TRADE » désignait un groupe hétérogène de sujets formellement autonomes mais matériellement salariés car travaillant pour une seule entreprise. Il faut préciser que travail indépendant économiquement dépendant et fausse autonomie ne sont pas la même chose. Le premier fait référence aux nouvelles formes de travail résultant de l'externalisation et de la sous-traitance d'activités non stratégiques dans lesquelles le travailleur bénéficie de l'indépendance nécessaire, tandis que le second concept fait référence à une relation de travail dissimulée, dans laquelle est simulé un contrat civil ou

1 Ces données qui sont disponibles dans « Résumé des résultats : travailleurs indépendants, personnes physiques inscrites à la sécurité sociale » (www.mitramiss.gob.es) contrastent avec le chiffre de 187000 travailleurs indépendants qui travaillent principalement pour une entreprise selon I'Institut national de Statistiques (INE) aux mêmes dates et disponibles sur www.ine.es 
commercial privant le travailleur des avantages inhérents à son statut de salarié ${ }^{2}$ On a tenté de corriger certaines situations frauduleuses avec la publication de la loi 20/2007 du 11 juillet, réglementant le statut du travail autonome (LETA); mais le succès fut mince ${ }^{3}$, la doctrine considérant que "lorsque le législateur est averti de l'existence d'une fraude constante, il doit reconnaître l'échec de la réglementation $»^{4}$.

Deuxièmement, le choix législatif a été influencé par une vision réductrice du champ d'application du droit du travail tendant à mettre fin à " la tendance traditionnelle à l'expansion du droit du travail dans le but de couvrir toutes les situations de dépendance, que ce soit juridique ou économique ${ }^{5}$. S'agissant des travailleurs indépendants d'un point de vue organisationnel, et dépendants d'un point de vue économique, étant le client majoritaire ou principal, voire ayant une unique source de revenus, il était entendu que leur intégration dans l'environnement de travail subordonné poserait des problèmes d'adaptation à la législation du travail. Ainsi, la LETA place le TRADE « sur un plateau en argent vers la non professionnalisation ... en lui octroyant un régime de garanties faibles et irréalistes ${ }^{6}$ pour accorder la préférence aux conditions d'application des dispositions au lieu de répondre au besoin de protection créé par la dépendance économique du travailleur vis-à-vis de l'entreprise cliente. Les opinions doctrinales sur la réglementation par la LETA de ce groupe de travailleurs sont disparates bien qu'elles soient polarisées entre d'un côté ceux qui voient comme un succès le fait de situer TRADE dans le cadre du travail autonome, et ceux qui estiment qu'il favorise la marchandisation d'une relation alors qu'il s'agit clairement d'une relation de travail ${ }^{7}$, l'indépendance technique et fonctionnelle du prestataire de services prévalant sur la dépendance économique caractéristique du travail salarié.

2 Pour une analyse de la frontière entre faux TRADE et travailleur salarié dans la doctrine espagnole, voir C. Molina, "¿Qué fue de los TRA(U)DES? El evanescente mundo de los (¿falsos?) autónomos", Revista Trabajo y Seguridad Social, CEF, n 401-402, 2016, p. 145.

3 Les données officielles indiquent qu'il existe actuellement 150000 faux travailleurs indépendants dans l'économie espagnole. Les données contenues dans l'Enquête sur la population active (EPA) sont disponibles sur le lien : www. ine.es

4 L. López, "Start-ups y capitalismo de plataforma: renovación o adaptación de los presupuestos laborales" en L. López (dir.), M. Revuelta (coord.), Start-ups, emprendimiento, economía social y colaborativa. Un nuevo modelo de relaciones laborales, Navarra, Thomson Reuters Aranzadi, 2018, p. 106

5 J. Cruz, "El trabajo autónomo económicamente dependiente en España. Breve valoración de su impacto tras algunos años de aplicación", Documentación Laboral, n98, 2013, p. 22.

6 A. Desdentado, "El traje nuevo del emperador. Sobre la legislación simbólica en el Estatuto del Trabajo Autónomo", Revista de Derecho Social, n 44, 2008, p. 24 et 35. Dans le même sens, J. Cruz Villalón indique qu'« il n'est pas possible de considérer que le Statut du travail autonome, par le biais de la création de la figure du travailleur indépendant économiquement dépendant, réponde à un modèle de " professionnalisation " de la relation juridique susmentionnée. Au contraire, il entre essentiellement dans le champ de la législation civile, sans incorporation dans le champ de la législation du travail ». J. Cruz, "El trabajo autónomo económicamente dependiente en España...", ob. cit. p. 28.

7 P. Rivas, "Aspectos estructurales y primeras reflexiones sobre el Estatuto del Trabajo Autónomo", Revista Española de Derecho del Trabajo, n 136, 2007, p. 781. 
Malgré les divergences d'opinions, la LETA était la première norme du système juridique espagnol qui systématisait le régime juridique des travailleurs indépendants. Elle a été configurée comme une norme-cadre dans laquelle ont été dessinées les lignes de base de la réglementation juridique du travail autonome en général et de la dépendance économique en particulier. C'était aussi une norme ambitieuse lorsque l'on essayait de régler toutes les questions liées à la fourniture de services.

Depuis sa publication, trois modifications ont été apportées. La première, par la loi 36/2011 du 10 octobre, réglementant la compétence sociale, introduisait la reconnaissance judiciaire du statut TRADE. La seconde, par la loi 31/2015 du 9 septembre qui visait à promouvoir le travail indépendant en adoptant une série de mesures de portée différente. La dernière, la plus importante, est contenue dans la loi 6/2017 du 24 octobre et vise à réformer en profondeur certaines questions qui affectent le plus la réalité du travail indépendant ${ }^{8}$. Néanmoins, malgré les progrès réalisés, cette dernière réforme n'a pas satisfait aux principales revendications formulées par les Associations professionnelles de travailleurs indépendants.

\section{I - LA CONFIGURATION DU TRAVAILLEUR ÉCONOMIQUEMENT DÉPENDANT DANS LA LOI 20/2007 DU 11 JUILLET, RÉGULANT LE STATUT DU TRAVAIL INDÉPENDANT}

La LETA dans le traitement de la régulation du travail indépendant économiquement dépendant se concentre non seulement sur la nécessité objective de fournir une réponse juridique adéquate à un besoin collectif de protection similaire à celle des travailleurs salariés, mais elle vise également à faire disparaître la frontière entre les deux catégories de travailleurs en empêchant l'utilisation abusive de la figure du TRADE. Ainsi, il s'agit de distinguer les deux catégories de travailleurs en clarifiant à la fois leur situation professionnelle et leur condition économique.

En plaçant TRADE à mi-chemin entre le travail salarié et l'emploi indépendant, le législateur a dû choisir entre l'insérer dans le lieu de travail tout en reconnaissant certaines particularités de son statut, ou le placer en dehors des murs de l'entreprise en lui attribuant des garanties qui ne profiteraient pas au reste des travailleurs indépendants. Un choix de politique législative qui a finalement estimé plus approprié de ne pas étendre les frontières

8 De manière générale, la loi 6/2017 du 24 octobre, relative aux réformes urgentes du Travail autonome, introduit des modifications de la sécurité sociale qui affectent les cotisations à la Sécurité Sociale, l'incorporation des accidents du travail in itinere parmi les risques professionnelles ou le régime de surtaxe; consacre des avantages économiques pour la maternité et la paternité comparables à ceux dont bénéficient les travailleurs salariés; en termes d'embauche, encourage la constitution en société de membres de la famille, introduit une compatibilité entre la pension de retraite et le travail indépendant; insère la possibilité de déduire certaines dépenses liées à l'exercice de l'activité autonome. Pour une étude approfondie du système juridique du travail autonome après la loi 6/2017 Monereo Pérez et Vila Tierno (dir.), «El trabajo autónomo en el marco del Derecho del Trabajo y de la Seguridad Social. Estudio de su régimen jurídico », Granada, Comares, 2018. 
du travail salarié, y compris dans le cadre du travail indépendant à ceux qui exercent une activité économique ou professionnelle à but lucratif, de façon habituelle, personnelle, directe et principale pour le compte d'une personne physique ou morale, appelée client, dont il dépend économiquement en percevant au moins $75 \%$ du revenu du travail et du revenu des activités économiques ou professionnelles.

L'art. 11 de la LETA rassemble les éléments qui définissent cette figure ( $1^{\text {e }}$ paragraphe), ainsi qu'un certain nombre d'exigences qui la qualifient ( $2^{e}$ paragraphe) et qui servent à le distinguer du travailleur subordonné et du travailleur indépendant ordinaire ${ }^{9}$.

En vertu de la loi, sont travailleurs indépendants économiquement dépendants « ceux qui exercent une activité économique ou professionnelle à but lucratif, de façon habituelle, personnelle, directe et principale pour une personne physique ou morale, appelée client, dont il dépend économiquement pour en percevoir au moins $75 \%$ de leur revenu du travail et du revenu des activités économiques ou professionnelles ». Le préambule de la LETA qualifie le concept de TRADE de restrictif en définissant selon des critères objectifs les circonstances dans lesquelles l'activité est effectuée en dehors du cadre de l'organisation et de la gestion du client qui recrute le travailleur indépendant.

Ainsi il est nécessaire, d'abord, que le travailleur effectue le travail de façon personnelle, régulière, directe, sans extranéité et en indépendance juridique même si elle n'est pas économique, dont le revenu dépend d'au moins $75 \%$ d'un seul client. La dépendance économique du TRADE est, par conséquent, l'élément déterminant si bien qu'à défaut il n'est pas possible d'accéder au régime de protection établi dans le chapitre III de la LETA. Cette exigence a soulevé une controverse considérable en ce qui concerne sa quantification et sa forme d'accréditation. Tout d'abord, fixer le niveau de dépendance économique du travailleur indépendant à au moins $75 \%$ de leur revenu, consiste à fixer un plancher, ouvrant la possibilité d'un lien complet entre le TRADE et son client. En d'autres termes, le législateur admet que la dépendance économique du TRADE vis-à-vis de son client puisse atteindre $100 \%$ de ses revenus. Dans ces conditions, la figure du travailleur indépendant peut se diluer dans une proximité douteuse avec la relation de travail. Dans ce cas, la LETA aurait dû envisager la possibilité d'admettre la nature du caractère salarié de la relation pour que l'indépendance juridique ou technique du travailleur n'agisse pas comme un voile empêchant de révéler la vraie nature de la relation inter partes surtout lorsque la législation espagnole prévoit des relations de travail dans lesquelles existe un haut degré d'autonomie des travailleurs dans l'exercice de leur prestation ${ }^{10}$.

La dépendance économique est liée à une autre notion non moins importante : l'exclusivité. L'art. LETA 12.2 prévoit que le statut de dépendance ne peut tenir que par rapport à un seul client. Comme nous l'avons déjà signalé à d'autres occasions ${ }^{11}$, il y aurait eu moins de problèmes d'application si on avait retenu l'élément de l'exclusivité comme critère de la condition TRADE au lieu de l'élément quantitatif de la dépendance économique.

9 J. Cruz, "El trabajo autónomo económicamente dependiente en España...", op. cit. p. 25.

10 Tel est le cas de la relation particulière de travail des avocats dans les cabinets d'avocats (RD 1331/2006 du 17 novembre) ou la relation spéciale de travail des cadres supérieurs (RD 1382/1985 de $1^{\text {er }}$ août).

11 E. Guerrero, “La (¿acertada?) regulación del trabajador autónomo económicamente dependiente: análisis del artículo 11 del Estatuto del Trabajo Autónomo", Revista Aranzadi Social n 20, 2010 p.162. 
Le RD 197/2009 du 23 février qui développe la LETA en relation avec la formalisation du contrat, prévoit que le niveau de revenu du TRADE en relation avec le client est certifié par la documentation que les deux parties conviennent d'établir ou de tout mode de preuve valable en droit et, quoi qu'il en soit, par les déclarations mentionnées au contrat que le travailleur indépendant et le client doivent accomplir (art. 2, 3 et 5.2 RD 197/2009). En ce qui concerne les conditions de forme (article 12.1 LETA), le contrat est écrit bien que la doctrine judiciaire ait décidé que la forme du contrat avait un caractère déclaratif ou probant. L'absence de formalisation écrite n'empêche pas le TRADE de revendiquer la jouissance des droits qui lui sont accordés. Cependant, en vertu de l'art. 12.2 LETA, « le travailleur indépendant doit indiquer expressément dans le contrat son statut de personne dépendante sur le plan économique vis-à-vis du client qui l'engage ... ». C'est pourquoi, le TRADE est obligé de faire connaître au client la situation de dépendance dans laquelle il se trouve à son égard dans le but de la formaliser dans le contrat. Si le travailleur n'informe pas le client avec lequel il se lie de sa condition de dépendance économique, la nature du contrat serait à leur discrétion (question interdite par l'art. 1256 du Code civil) susceptible de créer un vice de consentement parce que le client aurait ignoré la nature réelle du contrat conclu. Pour éviter la nullité du contrat, la LETA impose au travailleur de communiquer sa situation de dépendance économique au client avec lequel il s'engage.

Cependant, pour être considéré comme un TRADE il ne suffit pas d'être une personne développant une activité économique ou professionnelle habituelle, personnelle et directe, en obtenant directement au moins $75 \%$ des revenus d'un unique client. La LETA impose d'autres conditions à défaut desquelles le travailleur est soumis au droit commun des contrats. Ces exigences spécifiques sont les suivantes:

- il ne devrait pas avoir à sa charge de travailleurs salariés, ni embaucher ou sous-traiter tout ou une partie de ses activités à des tiers. Le TRADE doit réaliser lui-même son activité économique et professionnelle, ce qui suppose un caractère personnel de la relation personnelle qui n'est pas requis s'agissant des travailleurs indépendants. La limitation concerne sa propre activité, qu'elle soit contractée avec le client dont elle dépend économiquement ou celle contractée avec d'autres clients. Cette exigence obéit au " profil sociologique que le législateur a retenu du TRADE : il ne veut pas d'employeur, même modeste, et commence donc par éliminer la possibilité d'être un employeur ${ }^{12}$. Le difficile équilibre entre cette exigence et les besoins de conciliation vie familiale/professionnelle a conduit à clarifier cette interdiction. La loi 31/2015 du 9 septembre a introduit la possibilité d'embaucher un salarié lorsque le TRADE se trouve dans certaines circonstances, permettant ainsi le maintien du contrat et évitant les dommages que l'interruption de l'activité pourrait causer au client lorsque cela est justifié par ces exigences de conciliation vie privée/vie professionnelle.

- il devrait gérer ses activités de manière différente des travailleurs qui travaillent pour le compte du client sur la base de contrats de travail. Autrement dit, l'activité développée par le TRADE doit être autonome, indépendante de l'exécution de son client. Avec cette exigence, il s'agit d'éviter les situations frauduleuses consistant à qualifier de travailleurs indépendants des travailleurs qui devraient être qualifiés de salariés, qui fournissent des services pour le compte du client principal sur le lieu de travail. Ce que la loi empêche, c'est la concurrence indifférenciée avec les salariés

12 C. Molero, "La configuración legal del autónomo dependiente: problemas y viabilidad", Revista Actualidad Laboral, n², 2008, p. 4. 
mais pas le fait qu'une même activité soit réalisée par des travailleurs indépendants différents, économiquement dépendants ou non ${ }^{13}$.

- il devrait disposer d'une infrastructure productive et de l'équipement nécessaire à l'exercice de l'activité indépendamment de son client, lorsque cette activité est importante sur le plan économique. La loi ne définit pas ce qu'il faut entendre par infrastructure ou quand elle a une pertinence économique ${ }^{14}$, la réponse judiciaire étant erratique. Ainsi, alors que dans certains cas, on a considéré que la contribution d'un véhicule réservée au montage de meubles par un travailleur n'avait aucune pertinence économique, dans d'autres, on considère que la moto et le téléphone portable utilisés par un livreur de Glovo sont des éléments qui, avec d'autres, emportent le statut de TRADE ${ }^{15}$.

- il devrait développer son activité selon ses propres critères d'organisation, sous réserve des indications techniques qu'il pourrait recevoir de son client. Avec cette précision la LETA réaffirme l'indépendance juridique et professionnelle du TRADE en évitant la soumission dans le domaine de la gestion et de l'organisation du client dont il dépend économiquement. Cette exigence ne porte pas atteinte aux indications techniques qu'il peut recevoir dans le cadre de son travail, qui sont destinées à l'exécution du service pour la satisfaction du client. Les nouvelles formes d'emploi issues de l'économie numérique ont rouvert le débat sur l'étendue de la subordination comme critère de distinction entre travail autonome et travail salarié, en tenant compte de la nécessité de reformuler un concept qui ne répond pas à la réalité économique actuelle ${ }^{16}$.

- il devrait percevoir une contre-prestation économique en fonction du résultat de l'activité, conformément à l'accord avec le client et en admettant le risque et la chance de cette activité.

Toutes ces exigences doivent, donc, être réunies pour que le travailleur indépendant et économiquement dépendant de son principal client pour au moins $75 \%$ de son revenu puisse être décrit comme un TRADE et puisse accéder au régime juridique plus protecteur que lui attribue la LETA. Dans l'ensemble, ces exigences réaffirment l'indépendance et l'absence d'aliénation du travailleur dans l'exercice de sa prestation. Cependant, les imperfections de cette réglementation posent de nombreux problèmes d'application qui, dix ans plus tard, mettent en cause l'objectif de protection poursuivi par la réglementation en faveur de cette catégorie de travailleurs.

13 J.Pérez, "El régimen profesional del trabajo autónomo económicamente dependiente: novedades legales y jurisprudenciales", Albacete, Bomarzo, 2016, p. 33.

14 STSJ Castilla y León (Burgos) 412/2017 de 22 de junio (rec. 375/2017).

15 Les décisions du Tribunal Social (Madrid) n²84/2018 du 3 septembre 2018 (rec 1353/2017) et n¹2/2019 du 11 janvier (rec 418/2018) sont les premières qui ont déclaré en Espagne qu'un travailleur numérique avait le statut de TRADE. Au contraire, la dernière décision du Tribunal Social (Madrid) n 53/2019 du 11 février a déclaré que la relation entre el travailleur et Glovo était une relation de travail salariée

16 Les salariés, dans bon nombre des nouvelles formes d'organisation du travail, jouissent d'autonomie dans la planification et l'exécution des tâches. Nous devons donc nous demander si, selon une approche sociologique, la distinction entre les statuts TRADE et salarié est durable. A. Riesco-Sanz, "Trabajo, independencia y subordinación", Revista Internacional de Sociología, vol. 74, n 1, 2016, p. 6. 


\section{II - CONTROVERSES AUTOUR DU CONCEPT JURIDIQUE DE TRADE}

Les conséquences des imperfections de la LETA se sont manifestées d'abord par la reconnaissance inter partes de la condition de TRADE. Dans un geste qui pourrait être décrit comme naïf, la rédaction initiale de la loi a consisté à établir une simple obligation du travailleur d'informer son client qu'il était économiquement dépendant à son égard, dans l'illusion qu'une telle condition serait spontanément acceptée. Tandis que la prolifération de cette catégorie de travailleurs obéissait à la volonté entrepreneuriale de rassembler flexibilité et efficacité économique hors du Droit du travail, le législateur voulait enfermer la prestation de services sur ce même terrain.

Suite à l'entrée en vigueur de la LETA la pratique a révélé des situations où le client, après la communication effectuée par le travailleur indépendant de son statut d'économiquement dépendant, parvenait à éluder cette information par diverses opérations pour retarder l'application du régime de protection de la LETA, comme par exemple en réduisant la charge de travail afin de ne pas atteindre le minimum de $75 \%$ du revenu ou tout simplement en ne renouvelant pas le contrat civil ou commercial à la date d'expiration. Afin d'éviter le premier de ces problèmes, la première modification de la LETA par la loi 36/2011 du 10 octobre a consisté à introduire l'article 11 bis pour corriger le « refus » du client principal de reconnaître la condition de TRADE en imposant cette reconnaissance par une procédure judiciaire.

Le nouvel article 11 bis établit que « le travailleur qui remplit les conditions énoncées dans l'article précédent peut demander à son client de formaliser un contrat de travailleur indépendant économiquement dépendant à travers une communication fiable. Dans le cas où le client refuse de formaliser le contrat ou quand un mois s'est écoulé depuis la communication et qu'il n'a pas été inscrit dans le contrat, le travailleur indépendant peut demander la reconnaissance du statut de travailleur indépendant économiquement dépendant devant les tribunaux du travail ». À cet égard, il convient de noter que l'exigence formelle de la communication par le TRADE à son client est considérée comme essentielle, car son défaut associé à l'ignorance de sa situation par la société, détermine la nature civile ou commerciale de la relation, et la soumet à la compétence des juridictions civiles ou commerciales, et non du travai ${ }^{17}$. Par conséquent, face à une telle communication, le refus du principal client de reconnaître la condition de TRADE oblige à promouvoir sa déclaration par procédure judiciaire. Toutefois, cette reconnaissance judiciaire de la condition de TRADE n'aura aucune incidence sur la relation contractuelle existant entre les parties dans la période précédant le moment où la décision du tribunal est communiquée au client. Autrement dit, depuis le début de la relation contractuelle jusqu'au moment où, effectivement, la condition de TRADE est déclarée, aux relations inter partis ne leur seront pas appliquées les règles professionnelles des dispositions plus protectrices du chapitre III de la LETA, dont la mise en œuvre effective est subordonnée à la rapidité avec laquelle le travailleur indépendant réagit pour demander la reconnaissance ju diciaire de son statut en cas d'inaction du principal client.

Il convient de noter que la condition de TRADE peut se produire non seulement à I'instant initial de la conclusion du contrat civil, commercial ou administratif, mais aussi au cours de son exécution. Dans le premier cas, au moment de la formalisation du contrat, le travailleur indépendant connaîtra les revenus à percevoir et communiquera à son client qu'il a le statut de dépendance économique à son égard. Dans le cas où la dépendance

17 Voir notamment STS du 6 octobre 2011 (RJ 2011/7335), STS-27 novembre 2012 (RJ 2013/363). 
économique surgirait au cours de la relation contractuelle, l'art. 12.3 LETA prescrit que la condition de TRADE n'affectera pas la relation juridique respectant « entièrement le contrat signé entre les deux parties jusqu'à son terme, à moins qu'ils n'arrivent à le modifier pour le mettre à jour des nouvelles conditions correspondant à un travailleur indépendant économiquement dépendant ». Leur justification peut être recherchée pour éviter une imposition forcée du nouveau régime juridique alors qu'ils étaient liés par une relation commune autonome ${ }^{18}$. Cette prévision représente à nouveau un report significatif dans l'application du régime professionnel approprié qui se fonde sur la volonté des parties.

Un deuxième problème d'application pratique a surgi à propos de l'exigence légale du caractère personnel de la relation de travail du TRADE. II serait nécessaire qu'une intervention directe interdise l'embauche de salariés, en conséquence de quoi, des droits ont été légalement reconnus à cette catégorie de travailleurs au terme de compromis. Tel est le cas de l'exercice du droit à la cessation de l'activité pour congé maternité ou congé paternité. II y a d'une part l'engagement de la continuité de la relation contractuelle qui justifie d'accorder la faculté au client de résilier le contrat sans indemnité lorsque cette interruption de la fourniture de services par le congé maternité ou paternité du TRADE entraîne un préjudice important (art. 16.3 LETA); et d'autre part, est reconnu le droit du TRADE de concilier son activité professionnelle avec sa vie personnelle et familiale (art. 4.2.g LETA). Cette lacune a été comblée par la loi 31/2015 du 9 septembre, dont le préambule reconnaît l'absence de protection en ce qui concerne la conciliation de la vie professionnelle et personnelle de ce groupe de professionnels.

Ainsi, la loi 31/2015 introduit une exception à l'interdiction générale du recrutement d'un salarié par le TRADE établie dans l'art. 11.1.a) de la LETA. Elle n'est pas applicable en cas de grossesse, d'allaitement d'un enfant de moins de neuf mois, des périodes de congé maternité, paternité, d'adoption ou pré-adoptif ou d'accueil permanent, de garde d'enfants de moins de sept ans ou d'un membre de la famille handicapé ou en état de dépendance prise en charge. Dans toutes ces circonstances, le TRADE peut embaucher un seul et unique salarié. Cependant, la solution proposée par le législateur dénature l'un des éléments déterminants de ce travailleur: la nature personnelle et directe de sa prestation. Permettre dans ces cas que le TRADE puisse embaucher le rapproche du travailleur indépendant commun qui peut exercer son activité via une simple gestion d'affaires.

II convient de noter en outre que cette équation n'est pas absolue. Ainsi, si le contrat conclu par le TRADE avec son "substitut » a son origine dans la prise en charge d'un enfant de moins de sept ans ou d'une situation de famille handicapé ou de dépendance elle aura lieu pour une durée équivalente à la réduction de l'activité par le travailleur, mais ne peut pas dépasser $75 \%$ de la journée d'un travailleur à temps plein comparable. Deux niveaux de protection concernant le droit à la conciliation ont bien été établis. Le premier correspond à des situations de maternité, de paternité, d'adoption, de personne prise en charge, de risque pendant la grossesse ou l'allaitement pour lequel le remplacement du TRADE face au client est complet, couvrant l'ensemble de la journée convenue contractuellement ${ }^{19}$. Pour ce qui est du deuxième niveau il correspond à des situations de prise en charge d'un enfant de moins de sept ans ou d'un membre de la famille handicapé ou en situation de dépendance pour lequel la substitution du TRADE ne peut avoir lieu que dans la limite de

18 Voir E. Guerrero, El trabajador autónomo dependiente económicamente: un estudio jurídico laboral, Valladolid, Lex Nova, 2012, p. 196.

19 La durée du travail devrait figurer dans le contrat d'activité conclu entre les parties (art. 4 RD 197/2009 du 23 février). 
$75 \%$ de la journée de travail. II s'agit donc d'une règlementation qui vise à promouvoir le droit de conciliation travail/vie des travailleurs indépendants même si elle contient des nuances importantes.

Enfin, grâce à l'embauche d'un salarié par le TRADE, on a également essayé de corriger ces situations où, face à une interruption justifiée de son activité pour cause de maternité, paternité ou incapacité temporaire, entre autres, le client prétendant subir un préjudice important pouvait résilier le contrat sans verser d'indemnité au TRADE. À cet égard, l'article 16.3 de la LETA déclare que les causes d'interruption de l'activité prévues dans les paragraphes précédents ne peuvent pas justifier la résiliation du contrat par le client... Cependant lorsque, dans les cas visés aux points d), e) et g) du paragraphe 1, l'interruption provoque un préjudice important pour le client qui paralyse ou perturbe le cours normal des affaires, pourra être considérée comme justifiée la résiliation du contrat...

En bref, la LETA établit une règle générale qui empêche de résilier le contrat dans certaines circonstances, de les considérer comme des raisons qui justifient la cessation d'activité par le TRADE, même si la possibilité de résilier le contrat est établie lorsque les causes d'interruption provoquent un préjudice important pour le client, au point de paralyser ou perturber le déroulement normal de son activité. Avec la nouvelle rédaction de la loi, il s'agit de rendre plus efficace la règle générale et d'empêcher le client de faire usage de l'exception et de résilier le contrat en autorisant le TRADE à poursuivre son activité avec un travailleur salarié en cas de maternité, de paternité, d'incapacité temporaire, etc. II faut noter que le texte dans sa version nouvelle ne précise pas le régime de responsabilité, les garanties et les obligations assumées par le TRADE comme employeur, à la fois contre le principal client et à l'égard du salarié.

\section{III - DÉLIMITATION INSUFFISANTE DU TRADE ET SON IMPACT SUR LA QUALIFICATION DES TRAVAILLEURS DU NUMÉRIQUE}

L'analyse montre que si elle est réussie, l'intention originale de la LETA d'offrir au TRADE une réglementation plus protectrice que celle que la législation espagnole propose aux travailleurs indépendants, une mauvaise délimitation du concept conduit à une évasion de ce groupe vers d'autres formes atypiques de prestation de services ${ }^{20}$. Si l'aspiration ultime de la LETA était la régularisation d'un grand groupe de travailleurs ayant migré dans cette zone frontière entre autonomie et subordination, la situation actuelle est certainement décourageante.

Les raisons de cet usage limité de la figure du TRADE se concentrent principalement sur deux aspects :

a) Le refus des entreprises de transformer une situation de pleine autonomie (travailleur indépendant ordinaire) en une situation de dépendance économique (TRADE) étant donné la proximité avec le travail salarié sur les droits reconnus par la LETA. Ainsi, bien qu'ils ne sont pas salariés, ils ont un statut juridique proche de celui des salariés dans des domaines tels que les horaires, les jours fériés, les cas de résiliation indemnisée, etc. ; ce sont donc des limitations imposées en matière contractuelle

20 Dans son rapport Non-standard employment around the world : understanding challenges, shaping prospects (2016), I'Organisation Internationale du Travail (OIT) a inclus les faux indépendants parmi les formes atypiques d'emploi (p.36). Ce document analyse les différentes formes d'emploi atypiques et formule des propositions pour promouvoir le travail décent, en particulier dans la gig economy. 
au principal client. Ce régime juridique plus protecteur est rejeté par les entreprises clientes au moment d'approcher le TRADE.

b) La LETA attribue à la juridiction sociale compétence pour tous les litiges découlant du contrat entre le TRADE et le client.Ainsi se produit le paradoxe de la reconnaissance d'un double régime pour ces travailleurs: a) en ce qui concerne la formalisation du contrat et la prestation de services, ils sont soumis à un système civil, commercial ou administratif en fonction de la nature du contrat, b) dans la résolution des litiges relatifs au contenu du contrat ils sont soumis à la juridiction sociale.

Le résultat est une figure sous-utilisée. Un autre effet se serait produit si le législateur espagnol avait choisi d'intégrer ce travail dans le domaine du travail salarié plutôt que de I'incorporer dans le domaine du travail indépendant. II y a plus d'éléments qui approchent le TRADE du salarié que du travailleur indépendant, en particulier compte tenu du fait que les nouvelles technologies et méthodes de travail façonnent un nouveau type de travailleur, plus indépendant dans l'organisation de son travail, plus absent de l'espace physique que constitue l'entreprise, et plus lié à une rémunération aux résultats, autant de caractéristiques en mesure de briser la distinction traditionnelle entre travail salarié et travail indépendant. Le trait commun à toutes les plateformes numériques est qu'elles annoncent leurs avantages en mettant l'accent sur la liberté de décision du travailleur sur le moment et la durée de fourniture de leurs services.

La généralisation du travail par le biais d'entreprises telles qu'Uber, Glovo, Amazon Mechanical Turk ou Deliveroo soulève de nouveaux défis en matière de qualification juridique. La numérisation de l'économie provoque une évasion du travail salarié et une revitalisation du travail indépendant. La raison réside dans la réduction des coûts et la plus grande flexibilité organisationnelle qu'elles offrent. L'autonomie, la coordination et la participation sont désormais les caractéristiques des nouvelles façons de travailler et vont contre les postulats classiques du travail. Cependant, le TRADE ne semble pas être une représentation pouvant être largement reconnu dans le travail numérique. La doctrine est divisée à cet égard. Si une partie considère que le travail numérique doit être réglementé comme un travail salarié ${ }^{1}$, l'autre, dont nous faisons partie, préfère le cadre du travail indépendant économiquement dépendant ${ }^{22}$.

21 Y. Sánchez-Urán, Economía de plataformas digitales y servicios compuestos. El impacto en el Derecho, en especial, en el Derecho del Trabajo, La Ley Unión Europea, nº 57, 2018, p. 17 ; A. Ginés et S. Gálvez, Sharing economy vs Uber economy y las fronteras del Derecho del Trabajo en el nuevo entorno digital, Revista Indret $n^{\circ}$ 1, 2016, p. 18-26;I. Beltrán, Economía de plataformas (platform economy) y contrato de trabajo, disponible sur le site https://ignasibeltran.com/2018/02/07/ economia-de-las-plataformas-platform-economy-y-contrato-de-trabajo-ponencial

22 J. R. Mercader, El nuevo modelo de trabajo autónomo en la prestación de servicios a trevés de plataformas digitales, Diario La Ley, sección Ciberderecho, n 9, 11 juillet 2017, p. 11 ; I. Sagardoy et P. Núnez-Cortés, Economía colaborativa y relación laboral : ¿un binomio conflictivo?, Cuadernos de Pensamiento Político, janvier-mars, 2017, p. 98 ; S. Del Rey, Sobre el futuro del trabajo : modalidades de prestaciones de servicios y cambios tecnológicos, Revista IUSlabor n², 2017, p. 5 


\section{TRAVAIL AUTONOME ÉCONOMIQUEMENT DÉPENDANT}

Dans les décisions judiciaires ${ }^{23}$ rendues en Espagne, il n'existe pas de critère uniforme pour qualifier la relation contractuelle liant le travailleur et la plateforme. A titre d'exemple, alors que dans la décision du Tribunal social (SJS n 33 Madrid) du 3 septembre 2018, le travailleur de Glovo est classé comme salarié, dans la décision du Tribunal social (SJS Madrid $n^{\circ}$ 19) un autre travailleur de Glovo est qualifié de travailleur indépendant économiquement dépendant.

Sauf dans les analyses relatives á la plateforme numérique Glovo, on observe une préférence pour la qualification de travail salarié, excluant l'utilisation du TRADE comme valeur intermédiaire caractérisée par une configuration dans laquelle s'unissent l'indépendance organisationnelle et la subordination économique. Dans aucun des jugements susmentionnés dans lesquels la nature de la relation juridique est discutée, il n'est fait mention à cette forme possible de lien entre l'entreprise et le travailleur. II s'agit d'une omission au moins paradoxale étant donné que le TRADE permet au même travailleur d'être indépendant dans la prestation de services, d'avoir une protection sociale proche de celle offerte au salarié et une défense collective homogène par le biais des syndicats de travailleurs; d'où son attractivité comme formule juridique pour ces nouveaux services numériques.

23 Concernant Deliveroo (SJS Valence 244/2018 du 1er juin (rec. 633/2017), par rapport à Take Eat Easy (SJS Barcelone 213/2018 du 29 mai (rec. 652/2016) et Glovo (SJS Madrid 53/2019 du 11 février) ; dans un sens opposé (SJS Madrid 284/2018 du 3 septembre et SJS 12/2019 du 11 janvier) Disponible sur www.poderjudicial.es/search/indexAN.jsp.

\section{ESTHER GUERRERO VIZUETE}

Professeur de Droit du Travail et Chercheur postdoctoral, Faculté des Sciences Juridiques de I'Université Rovira et Virgili (Tarragona, Espagne).

Thèmes de recherche : Travail indépendant, polítiques de l'emploi, droit du travail.

\section{Publications:}

E. Guerrero Vizuete, "La digitalización del trabajo y su incidencia en los derechos colectivos de los trabajadores", in Moreno Gené, Torres Coronas, Belzunegui Eraso (coord.), Finding solutions to societal problems, Publicaciones URV, 2018.

E. Guerrero Vizuete, "La economía digital y los nuevos trabajadores: un marco contractual necesitado de delimitación", Revista Internacional y Comparada de Relaciones Laborales y Derecho del Empleo, $\mathrm{n}^{\circ} 1,2018$.

E. Guerrero Vizuete, El trabajador autónomo dependiente económicamente: un estudio jurídico laboral, Lex Nova, Valladolid, 2012. 